\begin{tabular}{lc}
\hline & Sharif University of Technology \\
SCIENTIA & Transactions B: Mechanical Engineering \\
IRAN ICA & www.scientiairanica.com \\
\hline
\end{tabular}

\title{
2D hierarchical heat transfer computational model of natural fiber bundle reinforced composite
}

\author{
H. Wang ${ }^{\mathrm{a}}$, Y. Xiao ${ }^{\mathrm{b}}$ and Q.-H. Qin ${ }^{\mathrm{b}, *}$ \\ a. Institute of Scientific and Engineering Computation, Henan University of Technology, Zhengzhou, 450001, China. \\ b. Research School of Engineering, Australian National University, Canberra, ACT 2601, Australia. \\ Received 13 September 2014; received in revised form 18 November 2014; accepted 12 April 2015
}

KEYWORDS
Hierarchical
composite;
Natural fiber bundle;
Lumen;
Heat conduction;
Finite element
method;
Thermal conductivity.

\section{Introduction}

As early as 3000 B.C., mud filled with plant stems has been used to construct buildings. This can be viewed as a prototype of modern natural fiber reinforced composites, which are an emerging area in composite science. Currently, natural fibers, including kenaf, hemp, bamboo fibers etc., are environmental friendly low cost fibers compared to conventional man-made solid fibers like carbon and glass fibers, and have low density and high specific properties. More importantly, they are biodegradable. Due to the special features of natural fibers, composites reinforced with them, such

\footnotetext{
*. Corresponding author. Tel.: +61 261258274 ;

Fax: +61261250506

E-mail address: qinghua.qin@anu.edu.au (Q.-H. Qin)
}

\begin{abstract}
In this paper, a two-dimensional (2D) hierarchical computational model was of small lumens, which hints that the composite, consisting of matrix and natural fiber olume Element (RVE) of the composite, with fibers arranged periodically, was taken into consideration and equivalent models were converted from differently scaled RVEs by a procedure. Subsequently, numerical simulation of the heat-transfer thermal conductivity of each model was obtained numerically. To verify the developed
\end{abstract} (C) 2016 Sharif University of Technology. All rights reserved. as natural fiber reinforced polymer/cement composites, have been viewed as green and environmental friendly materials and, thus, have been widely accepted for engineering applications [1-3]. As one of the inherent characteristics of natural fiber is its microstructure, its excellent thermal insulating property has shown great potential for the development of new green and energysaving composites. It was suggested that the moisture content, thickness, internal air cavities and surface structuring of bark can significantly affect flame retardancy and heat insulation of wood [4]. Liu et al. revealed that natural hemp fibers, consisting of cellulose or lumens, can present lower thermal conductivity [5]. Considering the high and increasing cost of energy harvesting and the ecological hazards of over consumption of energy, studying the thermal properties of natural fiber or composites filled with it is becoming an ever 
important issue. Current research includes experimental investigation of the influence of fiber content on the mechanical and thermal properties of kenaf fiber reinforced thermoplastic polyurethane composites [6], pineapple leaf fiber reinforced composites [7], hemp fiber reinforced composites [8], finite element simulation and analytic modelling the transverse thermal conductivity of Manila hemp fiber in solid regions [5], PLA-bamboo fiber composites [9], the temperature profile and curing behavior of hemp fiber/thermoset composite during the molding process [10], and the effect of the microstructure of natural fiber on the transverse thermal conductivity of unidirectional composite filled with natural fibers [11]. Additionally, Srinivasan et al. tested the mechanical and thermal performance of polymer composites filled with banana and flax fibers, whose cellulose content, respectively, are $63 \%$ and $71 \%$ [12]. Zheng numerically investigated the anisotropic thermal conductivity of natural fiber bundles with lumens, and the results showed that the large number of lumens in the fiber bundle can induce approximate isotropy [13]. All the work mentioned above is of great benefit regarding the understanding of the thermal transfer mechanism in natural fiber. However, these research works considered the fiber reinforced composite as a whole, or only studied the fiber microstructure itself. The answer to questions as to how the microstructure affects thermal conduction of bulk composite properties is yet unknown, which is critical in guiding the design of natural fiber filled composites with desirable thermal properties.

Hierarchical structures are exhibited in many natural and man-made materials. Analysis based on different hierarchical levels is expected to give a deep understanding of the relationship between microstructures and the physical properties of composites. The hierarchical order of a structure or a material may be regarded as the number of levels of scale with a recognized structure [14]. At each level of the structural hierarchy, one may model the material as a continuum for the purpose of analysis. Such an assumption is warranted if the structure size at each level of the hierarchy is significantly different. Taking hemp fiber bundle reinforced composites, as an example, we study the bulk thermal properties of the composites affected by the inherent microstructure of fiber bundles via hierarchical structuring. In such a natural fiber bundle, there are a large number of lumens filled with air in their transverse directions (see the cross-section morphology of the hemp fiber bundle in [5] for details). The thermal properties of the natural fiber bundle may vary considerably depending on the volume and size of the lumens and the thermal property of the solid region encircling the lumen walls. Figure 1 shows a schematic illustration of a three-level hierarchy composite model, with each natural fiber

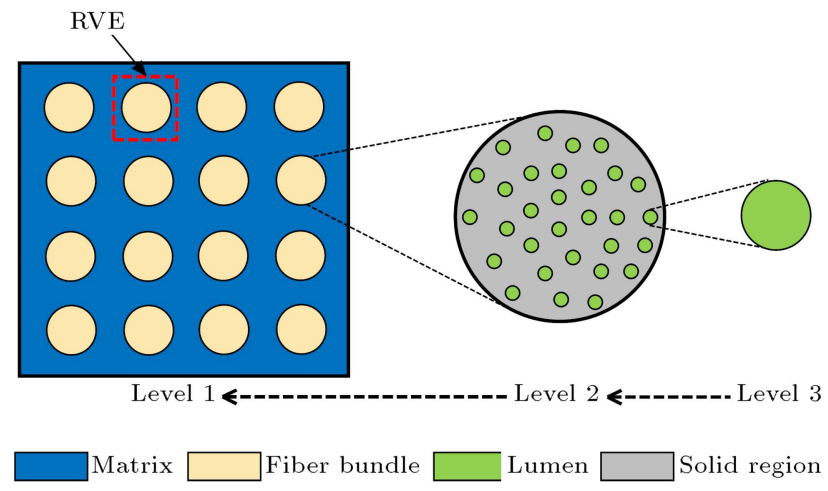

Figure 1. Schematic view of three-level hierarchy model of doubly periodic natural fiber bundle reinforced composites.

bundle consisting of lumens and a solid region. It is observed that the large-scale fiber bundle (i.e. about $200 \mu \mathrm{m}$ for a hemp fiber bundle) is filled with many small-scale lumens (i.e. about $16.5 \mu \mathrm{m}$ for lumen) in the solid region [5]. As such, an entire fiber bundle is regarded as a composite consisting of lumen fillers and a solid region phase. This allows us to analyze the thermal property hierarchically and use equivalency to bridge models between different scales. In this paper, a 2D hierarchical finite element model of a natural fiber bundle composite is established to investigate the influences of the microstructure of natural fiber bundles to the bulk thermal property of the composite [15]. A computational model is also employed to determine the optional interrelationship between the thermal properties of the solid region and the fiber bundle for the purpose of verification. Heat transfer in the natural fiber bundle reinforced composite is analyzed based on the Finite Element Method (FEM) [16,17], implemented by the commercial software ABAQUS. To verify the present composite model, the effects of the thermal property of the solid region phase on the equivalent thermal property of the fiber bundle are analyzed using the finite element model [18-20]. Then, an optional interrelationship of thermal properties between them is established by a curve fitting technique [21] to give a fast and accurate prediction of the material properties of both the fiber bundle and the composite as a whole. Further, a sensitivity analysis is conducted to investigate how value changes of important parameters, including thermal conductivity and volume fraction of the constituents, can affect the overall thermal properties of the composite.

\section{2D hierarchical finite element model of natural fiber bundle reinforced composite}

When the composite of interest has a doubly periodic distribution of fibers or fiber bundles, a unit Representative Volume Element (RVE) or cell of the composite 


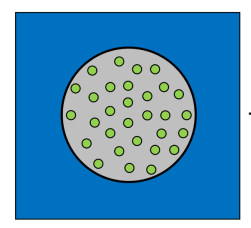

(a)

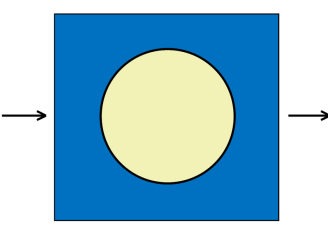

(b)

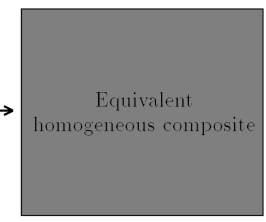

(c)
Figure 2. A two-step homogenized procedure for the natural fiber bundle reinforced composite: (a) Original RVE; (b) first equivalent RVE; and (c) second equivalent RVE.

can be adopted as the basic element. Then, two equivalent models in different size scales are converted by two-step homogenized procedures for heat-transfer analysis. The fiber bundle is assumed to be embedded into a square matrix phase like polymer, concrete, etc., to form a RVE composite system, as shown in Figure 2(a), including the matrix phase, lumen phase and solid region phase. Adjacent material phases are assumed to be bonded perfectly. In this schema, the fiber bundle is usually long enough in comparison to its cross section dimensions, so that the model is assumed to be infinite in the longitudinal direction. Moreover, theoretically, the distribution of the lumens will cause the anisotropy of the composite under consideration. In fact, the anisotropy of the composite becomes weak with the increasing number of lumens [5]. This means that when the number of lumens is large enough, the effect of the distribution of lumens tends to be isotropy. Here, we assume an isotropic thermal property of the fiber bundle for the case with a large number of lumens.

A two-step homogenization procedure is used here for thermal property calculation of the composite model, as shown in Figure 2. Firstly, the fiber bundle, consisting of large number of lumens, is homogenized to produce an equivalent material phase (see Figure 2(b)). This material phase is then considered as a reinforcement of the matrix in the transverse direction. The homogenization of this new composite model, as shown in Figure 2(b), gives the final equivalent homogeneous material phase, as displayed in Figure 2(c).

In order to determine the effective thermal property of the composite, proper boundary conditions should be prescribed to the RVE, as has been undertaken by many researchers in the analysis of heterogeneous materials [22-27]. Islam and Pramila [23] suggested that in heat-transfer analysis, the prescribed temperature boundary conditions on the parallel boundaries of the rectangular RVE can produce the most accurate results up to a relatively high fiber volume fraction. Thus, in the computing model displayed in Figure 3, two different temperatures, $T_{1}$ and $T_{0}$, are specified on its vertical edges, and the remaining top and bottom edges are assumed to be insulation. It is assumed that $T_{1}>T_{0}$, so that the horizontal heat flux loaded on the left side face (data-

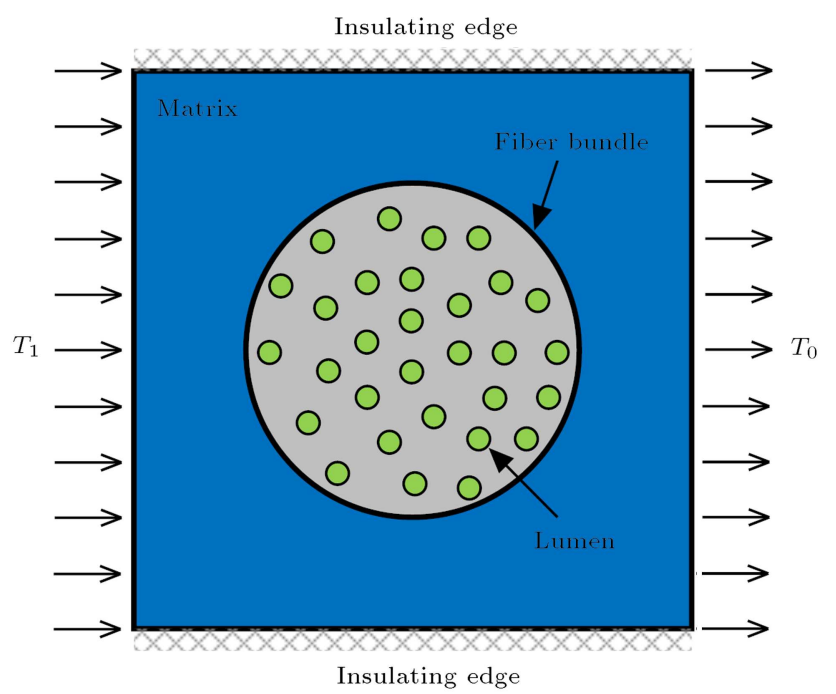

Figure 3. Schematic illustration of a square cell embedded with natural fiber bundle containing lumens.

collection surface) is positive. For instance, in practical computation, we can let $T_{1}=20^{\circ} \mathrm{C}$ and $T_{1}=0^{\circ} \mathrm{C}$.

According to Fourier's law of heat transfer in isotropic media, we have:

$$
q_{1}=-k \frac{\partial T}{\partial x}, \quad q_{2}=-k \frac{\partial T}{\partial y} .
$$

The effective thermal conductivity of the composite, $k_{e}$, can then be evaluated by $[23,28]$ :

$$
k_{e}=\frac{Q L}{\left(T_{1}-T_{0}\right)} .
$$

In Eqs. (1) and (2), $T$ is the temperature change in the Cartesian coordinate system, $x-y$, and $k$ is the thermal conductivity of the isotropic material. $Q$ stands for the average value of the heat flux component, $q_{1}$, loaded on the left side face of the cell, which can be evaluated by trapezoidal numerical integration. $L$ is the side length of the cell.

In our analysis, the side length of the RVE or cell used is assumed to be 1, which is a normalized length. Furthermore, the volume fraction of the lumens to the fiber bundle is assumed to be a constant of $30.87 \%$, which is an actual experimental value of the Manila hemp fiber bundle [5]. The volume fraction of fiber bundle to composite is initially set to be a constant of $50 \%$, a relatively high volume concentration, unless otherwise specified. The thermal conductivity of the matrix is taken to be $0.42 \mathrm{~W} /(\mathrm{mK})$, which corresponds to the polymer matrix [8]. According to the known research results [4], the anisotropy of the fiber bundle becomes smaller as the number of lumens in it increases, and then, the composite tends to isotropy. Here, since there is no available data on the number of lumens in a fiber bundle in the literature, 
it is assumed to be 106, which is close to the actual dispersion of lumens in the fiber bundle (see the crosssection morphology of the hemp fiber bundle in [4] for details) and is also enough to produce the isotropic thermal property of the fiber bundle and the composite. Besides, the thermal conductivities of a composite, matrix and solid region are, respectively, normalized, with respect to those of lumen, that is, $0.026 \mathrm{~W} /(\mathrm{mK})$, in the following post-processing of numerical results. Finally, to simplify the analysis, symbols $k_{m}, k_{f b}, k_{s}$ and $k_{l}$ denote, respectively, the thermal conductivity of the matrix, the equivalent fiber bundle, the solid region and lumen, and $v_{f b}$ and $v_{l}$, respectively, stand for the volume fraction of the fiber bundle and the lumen.

\section{Numerical experiments}

\subsection{Verification of the present computational composite model}

As mentioned in the introduction, two composite models, including the original RVE and the first equivalent RVE, are here investigated, respectively, using the finite element method, with which the bulk thermal conductivities of composites are calculated. Then, an optional relationship of thermal conductivity between the solid region and the fiber bundle is derived by curved fitting to verify the present computational composite model.

\subsubsection{Computational analysis of the original composite model}

To conduct finite element analysis, the original composite model with 106 internal lumens, as shown in Figure 4(a), is discretized by a total of 51680 quadratic elements, with 155841 nodes (see Figure 4(b)), generated by ABAQUS. In order to determine the dependence of the thermal conductivity of the composite on that of the solid region, it is assumed that the normalized thermal conductivity of the solid region changes in the range $[1,10]$, which corresponds to the variation from poorly conducting to highly conducting. By means of finite element analysis, distribution of the horizontal

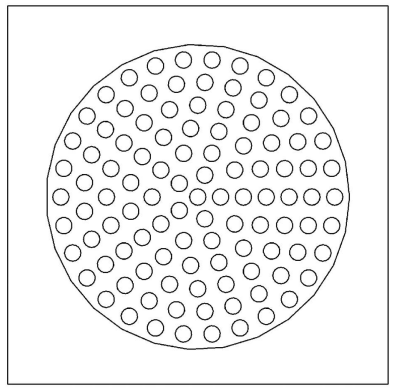

(a)

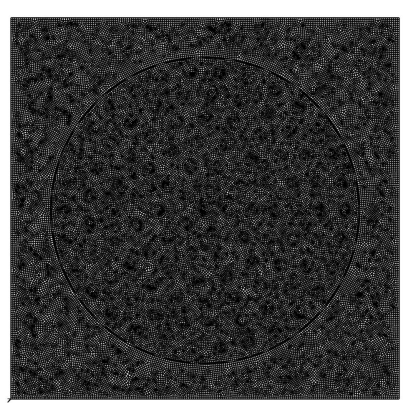

(b)
Figure 4. Finite element model of RVE of natural fiber bundle composites including 106 lumens: (a) Computational domain; and (b) computational mesh. heat flux component, $q_{1}$, on the data-collection surface can be determined. Then, its corresponding average value, $Q$, can be evaluated by trapezoidal numerical integration for each specific value of the normalized thermal conductivity of the solid region. Subsequently, the overall thermal conductivity of the composite is obtained using Eq. (2).

From the computational model, the contour plots of normalized temperature and horizontal heat flux components in the entire composite domain under consideration are, respectively, obtained and displayed in Figures 5 and 6 (for the case of $k_{s} / k_{l}=1$ and $k_{s} / k_{l}=5$ ) to demonstrate the effect of thermal conductivity in the solid region on the heat transfer performance in the composite. It is obvious that heat is transferred into the fiber bundle from left to right through thermal conduction. When $k_{s} / k_{l}=1$, the thermal conductivity of the solid region and lumen becomes identical. Thus, just a smaller portion of heat passes through the fiber bundle, while a greater portion of it passes around the fiber bundle, due to the low thermal conductivity of lumen. However, for the case $k_{s} / k_{l}=5$, the solid region has a larger thermal conduction capacity than that of the lumen, so, more heat flows into the fiber bundle and passes through the solid region. The mismatch of thermal property between the solid region and the lumen significantly increase the quantity of the heat flux component in the fiber bundle. Besides, in either case, the process of heat transfer in the composite becomes longer and more complicated, leading to reduction in the thermal properties of the composite. Furthermore, Figure 7 displays the variation of the normalized effective thermal conductivity of the composite in terms of the thermal conductivity of the solid region. Results in Figure 7 show that the simulated effective thermal conductivity of the composite increases with an increase in the value of the thermal conductivity of the solid region. Simultaneously, it is obvious that variation of the overall thermal conductivity of the composite exhibits slight nonlinearity. To model this change, we use a quadratic polynomial fitting curve:

$$
\frac{k_{e}}{k_{l}}=-0.008432\left(\frac{k_{s}}{k_{l}}\right)^{2}+0.5236\left(\frac{k_{s}}{k_{l}}\right)+5.673 .
$$

This is used to establish the approximated relationship of thermal conductivity between the solid region and the fiber bundle.

\subsubsection{Computational analysis of the first equivalent composite model}

Here, the first equivalent composite model, shown in Figure 8(a), is taken into consideration to investigate the effect of the equivalent fiber bundle on the composite. In the model, the practical fiber bundle is homogenized as a homogeneous material 


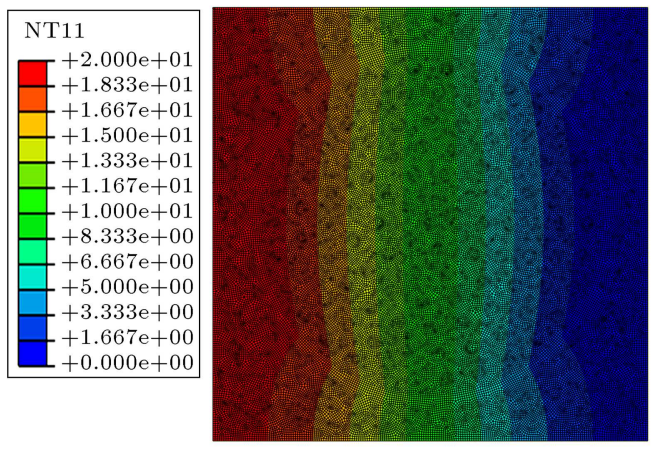

(a) $k_{s} / k_{l}=1$

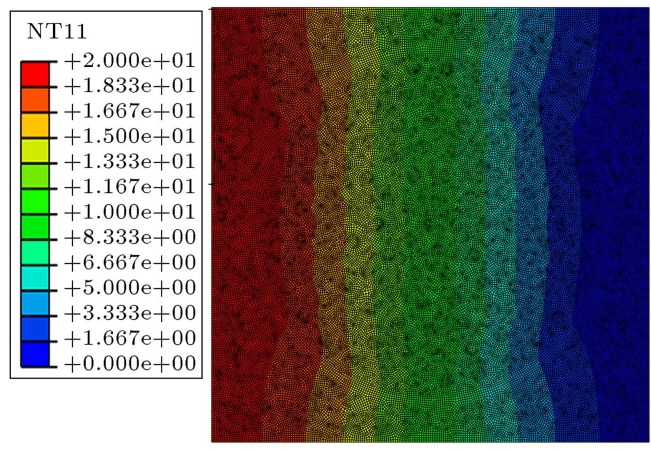

(b) $k_{s} / k_{l}=5$

Figure 5. Distribution of temperature in the computational domain.

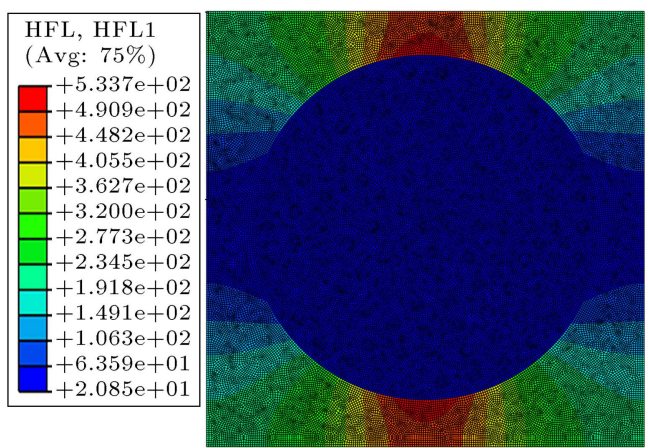

(a) $k_{s} / k_{l}=1$

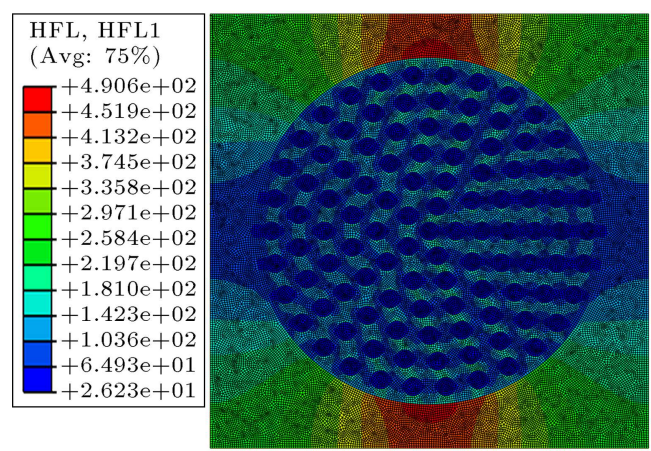

(b) $k_{s} / k_{l}=5$

Figure 6. Distribution of horizontal heat flux component in the computational domain.

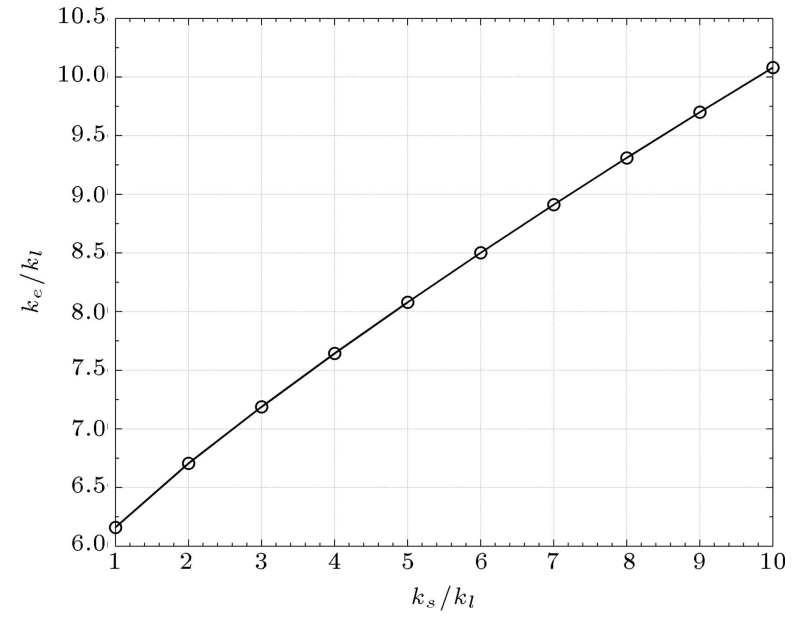

Figure 7. Variation of the effective thermal conductivity of the composite against that of the solid region.

with equivalent material property, and is replaced with a solid fiber of the same size for computation, as shown in Figure 2(b). The same boundary conditions as those applied in the original composite model, as shown in Figure 3, are employed. To carry out finite element simulation, 12247 quadratic quadrilateral heat transfer elements generated by ABAQUS are used to discretize the first equivalent composite domain. To investigate the variation of the thermal property of the composite caused by the homogenized fiber bundle, we

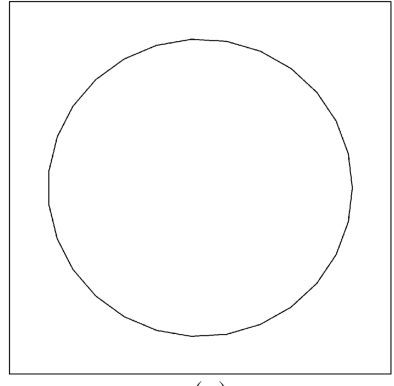

(a)

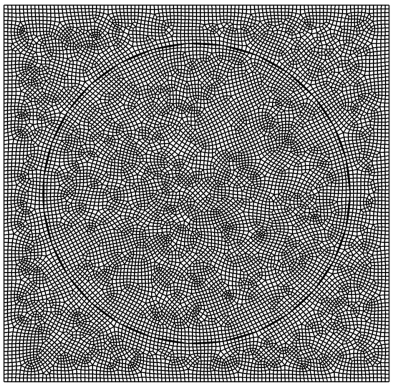

(b)
Figure 8. Finite element model of the first equivalent RVE: (a) Computational domain; and (b) computational mesh.

assume that the normalized thermal conductivity of the homogenized fiber bundle changes in the interval $[1,10]$. Thus, we can obtain a curve to describe the dependence between the equivalent fiber bundle and the composite. Numerical results in Figure 9 show that the simulated thermal conductivity of the composite increases with an increase in the value of the thermal conductivity of the equivalent fiber bundle, as expected. Also, a weak nonlinear variation is observed in Figure 9. Fitting the numerical results in Figure 9 by polynomial approximation yields:

$$
\frac{k_{e}}{k_{l}}=-0.01486\left(\frac{k_{f b}}{k_{l}}\right)^{2}+0.8932\left(\frac{k_{f b}}{k_{l}}\right)+5.301 .
$$




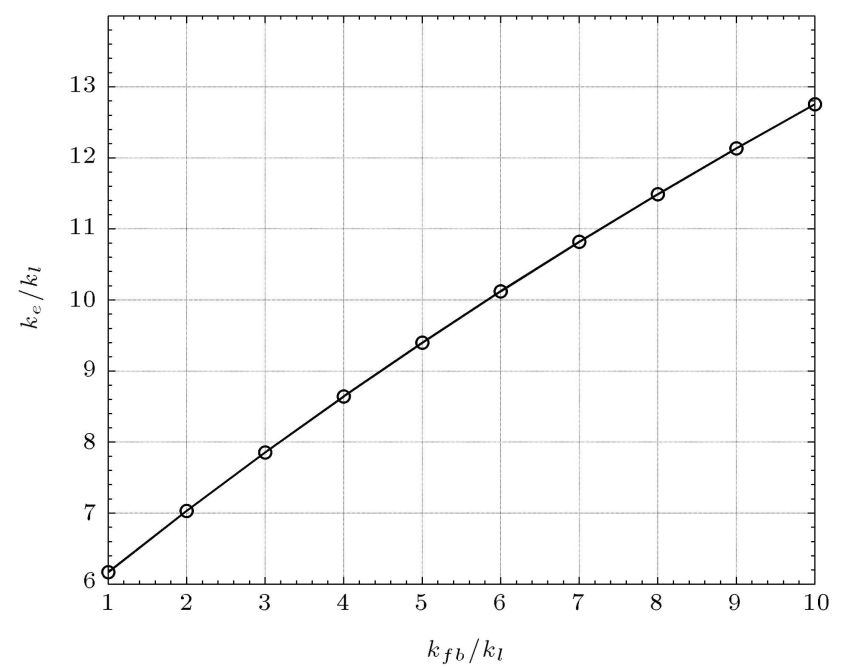

Figure 9. Variation of the effective thermal conductivity of the composite against that of the equivalent fiber bundle.

\subsubsection{Relationship of thermal conductivity between the solid region and the fiber bundle}

The equivalence of the two composite models, shown, respectively, in Figures 4(a) and 7, requires the same effective thermal conductivity of the composite under the same boundary conditions. Therefore, combining Eqs. (3) and (4), one can get an optional relationship of thermal conductivity of the fiber bundle and the solid region, that is:

$$
\begin{aligned}
& -0.01486\left(\frac{k_{f b}}{k_{l}}\right)^{2}+0.8932\left(\frac{k_{f b}}{k_{l}}\right) \\
& =-0.008432\left(\frac{k_{s}}{k_{l}}\right)^{2}+0.5236\left(\frac{k_{s}}{k_{l}}\right)+0.372,
\end{aligned}
$$

from which, the variation of $k_{s}$, with respect to $k_{f b}$, is plotted in Figure 10.

To verify the obtained relation of thermal conductivities between the solid region and the fiber bundle, an analytical solution, taken from the HasselmanJohnson's model $[5,29]$, is employed for comparison. Here, the analytical expression of the thermal conductivity of the solid region, $k_{s}$, with respect to that of the fiber bundle, $k_{f b}$, is written as:

$$
\frac{k_{s}}{k_{l}}=0.9465\left(\frac{k_{f b}}{k_{l}}-1\right)+\sqrt{0.8960\left(\frac{k_{f b}}{k_{l}}-1\right)^{2}+\frac{k_{f b}}{k_{l}}}
$$

with $v_{l}=30.87 \%$. Note that Eq. (6) is different from that in reference [5]. For the purpose of comparison, we plot Eq. (6) in Figure 10. Especially, if $k_{f b}$ is $0.115 \mathrm{~W} /(\mathrm{mK})[5,8] ; k_{s}$ can be calculated from Eq. (6) and has value of $0.1847 \mathrm{~W} /(\mathrm{mK})$.

In Figure 10, it is observed that there is good agreement between the quadratic fitting curve and

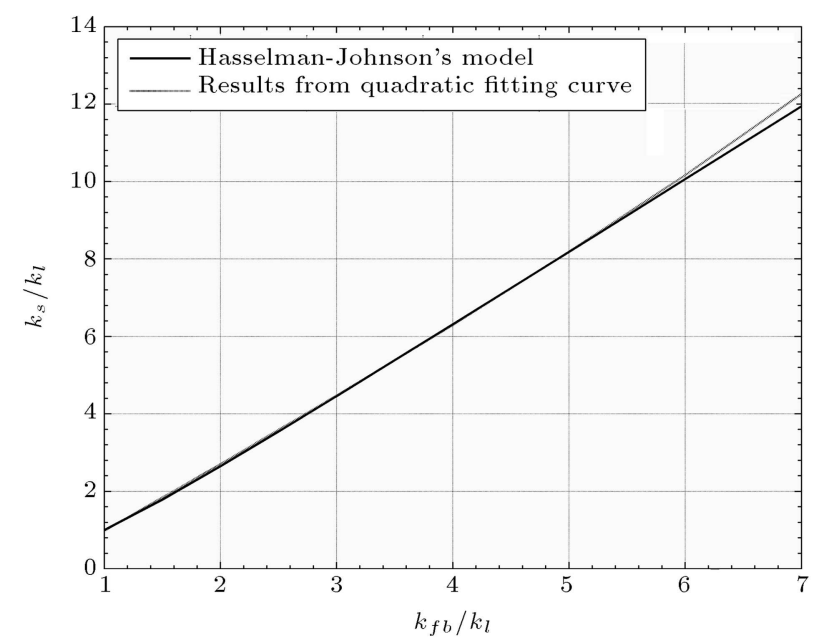

Figure 10. Approximated relation of thermal conductivities of the natural fiber bundle against the fiber solid region.

the theoretical curve. For example, when $k_{f b}=$ $0.115 \mathrm{~W} /(\mathrm{mK}), k_{s}$ from the fitting curve Eq. (5) is $0.1843 \mathrm{~W} /(\mathrm{mK})$, which has a relative deviation of $0.21 \%$ compared to the theoretical solution of $0.1847 \mathrm{~W} /(\mathrm{mK})$ (obtained from Eq. (6)). In research work [5], the evaluated value of $k_{s}$ is $0.1849 \mathrm{~W} /(\mathrm{mK})$. Therefore, the present computational composite model on the fiber bundle, including numbered lumens, has been verified and the obtained quadratic relationship between the thermal conductivity of the solid region and the fiber bundle can be used to estimate the thermal conductivity of the natural fiber or the solid region, if one of them is known.

Besides, from Figure 10, it is clear to see that the existence of lumens significantly weakens heat transfer in the fiber bundle, and then decreases the thermal conductivity of the equivalent fiber bundle, $k_{f b}$, in the first equivalent RVE. As a result, $k_{f b}$ must be less than $k_{s}$, significantly, which can be seen from Figure 10.

\subsection{Sensitivity analysis}

A sensitivity analysis is carried out in this section. The purpose of a sensitivity analysis is to identify individual or a combination of parameters that may affect the effective thermal conductivity of the composites. This is important since accurate prediction of the effective thermal conductivity of composites may significantly benefit their design.

The parameters examined here include the volume fraction of the fiber bundle to the matrix, the volume fraction of the lumen to the fiber bundle, and the thermal conductivity of the matrix. To investigate how changes in each of these parameters may affect the effective thermal conductivity of the composite, serial values that are different from practical values are considered, i.e. some values are higher than the practical value and others may be lower. 


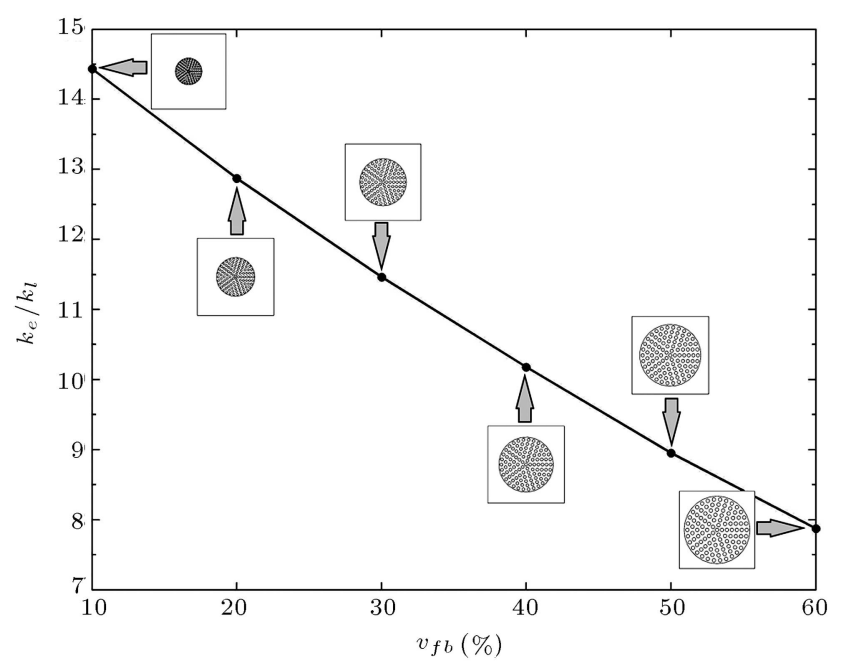

Figure 11. Variation of the effective thermal conductivity of the composite against the volume fraction of the fiber bundle.

\subsubsection{Effect of volume fraction of the fiber bundle}

Let us begin by considering the influence of the volume fraction of the fiber bundle on the effective thermal conductivity of the composite. In the analysis, the volume fraction of the lumen to the fiber bundle is assumed to be a constant of $30.84 \%$. Figure 11 presents the variation of the normalized thermal conductivity of the composite, $k_{e} / k_{l}$, in terms of the volume fraction of the fiber bundle, $v_{f b}$. It can be seen that there is a considerable decrease of $k_{e} / k_{l}$ when $v_{f b}$ increases. The average value of the decrease of $k_{e} / k_{l}$ is 1.312 for every $10 \%$ increase in the value of $v_{f b}$.

\subsubsection{Effect of lumen volume fraction to the fiber bundle}

The influence of the volume fraction of the lumen on the effective thermal conductivity of the composite is investigated in this section. In the analysis, the volume fraction of the fiber bundle to the composite is assumed to be $50 \%$. Alteration of the volume fraction of the lumen is fulfilled by changing the radius of the lumen. Figure 12 displays the variation of the normalized thermal conductivity of the composite, $k_{e} / k_{l}$, in terms of the volume fraction of the lumen, $v_{l}$. It can also be seen that there is a considerable decrease of $k_{e} / k_{l}$ with an increase of $v_{l}$. In this case, the volume fraction of the lumen increases by $10 \%$, and the normalized effective thermal conductivity will decrease about 0.538. In contrast to the volume fraction of the fiber bundle, the change of the volume fraction of the lumen has less influence on the effective thermal property of the composite.

\subsubsection{Effect of thermal conductivity of the matrix}

Finally, the effect of thermal conductivity of the matrix on the effective thermal property of the composite is investigated and the numerical results are displayed in

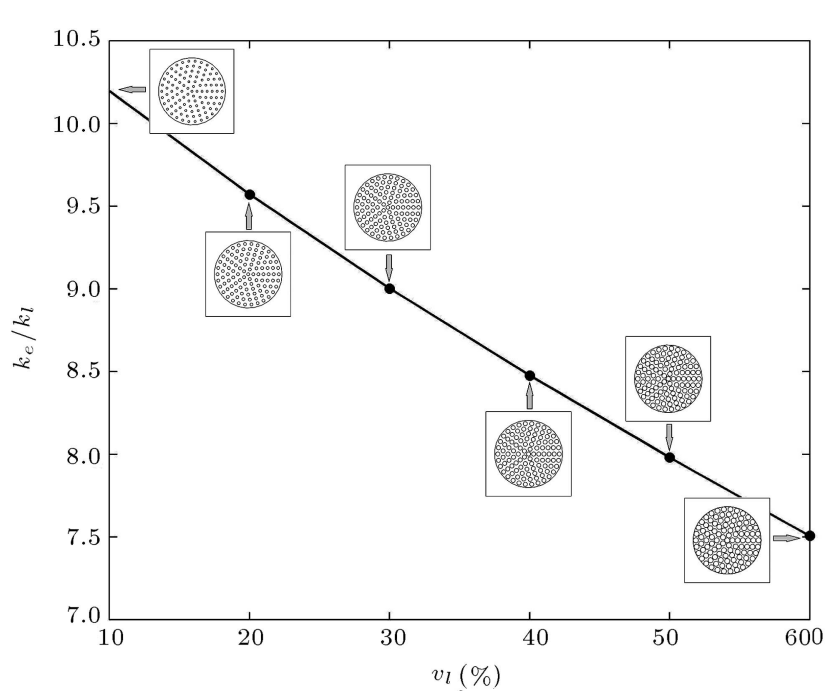

Figure 12. Variation of the effective thermal conductivity of the composite against the volume fraction of the lumen.

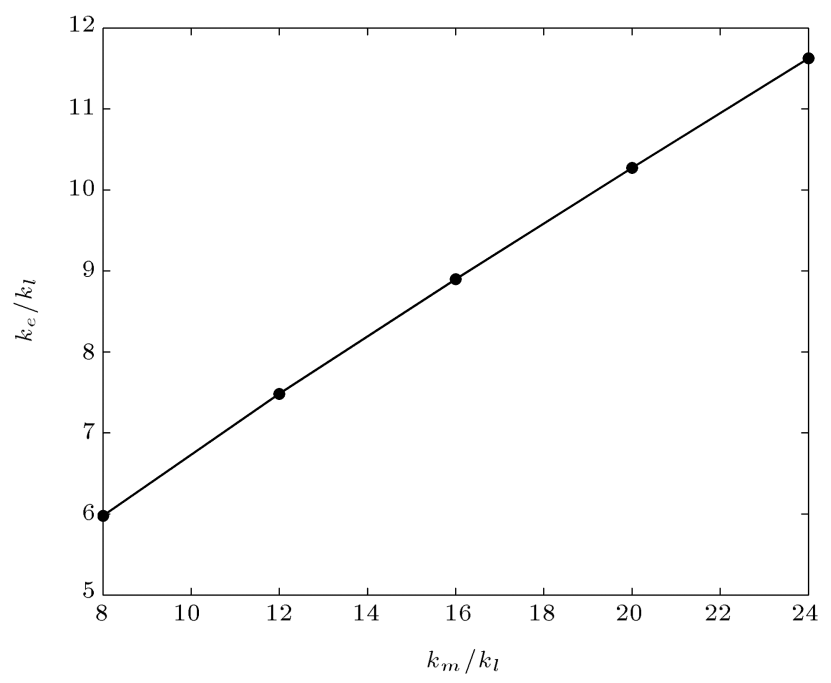

Figure 13. Variation of the effective thermal conductivity of the composite against the thermal conductivity of the matrix.

Figure 13. In the computation, the volume fractions of the fiber bundle and the lumen are taken as $50 \%$ and $30.87 \%$, respectively. It can be seen from Figure 13 that there is a remarkable increase of $k_{e} / k_{l}$ with the increase of $k_{m} / k_{l}$, and when the normalized thermal conductivity of the matrix increases by 1 , the normalized effective thermal conductivity will increase about 0.353. Compared to the numerical results in Figure 7, which depict the effect of thermal conductivity of the solid region in the fiber bundle on the effective thermal conductivity of the composite, it is found that the solid region has a larger influence than the matrix on the effective thermal property of the composite. The main reason is that the larger volume fraction of the fiber bundle dominates thermal transfer behavior in the composite. 


\section{Conclusion}

In this paper, a 2D hierarchical computational model of the heat transfer of unidirectional composites filled with a natural fiber bundle was developed and then verified by comparison with the analytic HasselmanJohnson's results and other numerical results. Subsequently, a sensitivity analysis was conducted to investigate the effects of the thermal conductivity of the solid region and the matrix, and volume fractions of the fiber bundle and lumen on the entire thermal conductivity of the composite. Numerical results show that the bulk thermal property of the composite decreases with an increase in the volume friction of both the fiber bundle and the lumen. The volume fraction of the fiber bundle has a more significant influence on the effective thermal property of the composite than the volume fraction of the lumen. Moreover, the bulk thermal property of the composite increases with an increase in the thermal properties of both the matrix and the solid region. For the case of a larger volume fraction of fiber bundle, the increase of thermal conductivity of the solid region produces the larger effective thermal conductivity of the composite than that of the matrix.

Besides, the present computational method is also applicable for evaluating the thermal conductivity of composites filled with other bundled natural fibers containing a large number of lumens, such as kenaf core fiber.

\section{Acknowledgements}

The research work is partially supported by the Natural Science Foundation of China (Grant no. 11102059; 11472099) and the Creative Talents Program of Universities in Henan Province (Grant no. 13HASTIT019).

\section{References}

1. Netravali, A.N., Huang, X.S. and Mizuta, K. "Advanced 'green' composites", Adv. Compos. Mater., 16(4), pp. 269-282 (2007).

2. Abdul Khalil, H.P.S., Bhat, I.U.H., Jawaid, M., Zaidon, A., Hermawan, D. and Hadi, Y.S. "Bamboo fibre reinforced biocomposites: A review", Mater. Des., 42, pp. 353-368 (2012).

3. Saheb, D.N. and Jog, J.P. "Natural fiber polymer composites: a review", Adv. Polymer Tech., 18(4), pp. 351-363 (1999).

4. Bauer, G., Speck, T., Blömer, J., Bertling, J. and Speck, O. "Insulation capability of the bark of trees with different fire adaptation", J. Mater. Sci., 45(21), pp. 5950-5959 (2010).

5. Liu, K., Takagi, H. and Yang, Z. "Evaluation of transverse thermal conductivity of Manila hemp fiber in solid region using theoretical method and finite element method", Mater. Des., 32(8), pp. 4586-4589 (2011).

6. El-Shekeil, Y.A., Sapuan, S.M., Abdan, K. and Zainudin, E.S. "Influence of fiber content on the mechanical and thermal properties of kenaf fiber reinforced thermoplastic polyurethane composites", Mater. Des., 40, pp. 299-303 (2012).

7. Mangal, R., Saxena, N., Sreekala, M., Thomas, S. and Singh, K. "Thermal properties of pineapple leaf fiber reinforced composites", Mater. Sci. Eng., 339(1), pp. 281-285 (2003).

8. Behzad, T. and Sain, M. "Measurement and prediction of thermal conductivity for hemp fiber reinforced composites", Polymer Eng. Sci., 47(7), pp. 977-983 (2007).

9. Takagi, H., Kako, S., Kusano, K. and Ousaka, A. "Thermal conductivity of PLA-bamboo fiber composites", Adv. Compos. Mater., 16(4), pp. 377-384 (2007).

10. Behzad, T. and Sain, M. "Finite element modeling of polymer curing in natural fiber reinforced composites", Compos. Sci. Tech., 67(7), pp. 1666-1673 (2007).

11. Liu, K., Takagi, H., Osugi, R. and Yang, Z. "Effect of physicochemical structure of natural fiber on transverse thermal conductivity of unidirectional abaca/bamboo fiber composites", Compos. Appl. Sci. Manuf., 43(8), pp. 1234-1241 (2012).

12. Srinivasan, V.S., Rajendra Boopathy, S., Sangeetha, D. and Vijaya Ramnath, B. "Evaluation of mechanical and thermal properties of banana-flax based natural fibre composite", Mater. Des., 60, pp. 620-627 (2014).

13. Zheng, G.Y. "Numerical investigation of characteristic of anisotropic thermal conductivity of natural fiber bundle with numbered lumens", Math. Probl. Eng., 2014, Article ID 506818, 8 pages (2014).

14. Lakes, R. "Materials with structural hierarchy", $N a$ ture, 361, pp. 511-515 (1993).

15. Kaw, A.K., Mechanics of Composite Materials, 2nd Edn., CRC Press, Boca Raton, US (2006).

16. Liang, X.P. and Wang, H., Applied Finite Element Analysis (in Chinese), Tsinghua University Press, Beijing, CN (2010).

17. Qin, Q.H. and Wang, H., Matlab and C Programming for Trefftz Finite Element Methods, CRC Press, Boca Raton, US (2009).

18. Qin, Q.H., The Trefftz Finite and Boundary Element Method, WIT Press, Southampton, UK (2000).

19. Qin, Q.H. "Variational formulations for TFEM of piezoelectricity", Int. J. Solid Struct., 40(23), pp. 6335-6346 (2003).

20. Dhanasekar, M., Han, J.J. and Qin, Q.H. "A hybridTrefftz element containing an elliptic hole", Finite Elem. Anal. Des., 42(14), pp. 1314-1323 (2006).

21. Kiusalaas, J., Numerical Methods in Engineering with MATLAB, Cambridge University Press, Cambridge, UK (2005). 
22. Qin, Q.H. and Wang, H. "Special circular hole elements for thermal analysis in cellular solids with multiple circular holes", Int. J. Comput. Methods, 10(4), p. 1350008 (2013).

23. Islam, M.R. and Pramila, A. "Thermal conductivity of fiber reinforced composites by the FEM", J. Compos. Mater., 33(18), pp. 1699-1715 (1999).

24. Qin, Q.H., Mai, Y.W. and Yu, S.W. "Effective moduli for thermopiezoelectric materials with microcracks", Int. J. Fract., 91(4), pp. 359-371 (1998).

25. Qin, Q.H. and Yang, Q.S., Macro-Micro Theory on Multifield Coupling Behaivor of Hetereogenous Materials, Higher Education Press and Springer, Beijing, CN (2008).

26. Yu, S.W. and Qin, Q.H. "Damage analysis of thermopiezoelectric properties: Part II. Effective crack model", Theor. Appl. Fract. Mech., 25(3), pp. 279-288 (1996).

27. Nemat-Nasser, S. and Hori, M., Micromechanics: Overall Properties of Heterogeneous Materials, Elsevier Science Publishers, Amsterdam, NL (1993).

28. Wang, H. and Qin, Q.H. "Special fiber elements for thermal analysis of fiber-reinforced composites", Eng. Computation, 28(8), pp. 1079-1097 (2011).

29. Hasselman, D. and Johnson, L.F. "Effective thermal conductivity of composites with interfacial thermal barrier resistance", J. Compos. Mater., 21(6), pp. 508515 (1987).

\section{Biographies}

Hui Wang was born in Luoyang City of China in 1976. He received his BS degree in Theoretical and Applied Mechanics from Lanzhou University, China, in 1999. He earned his MS degree from Dalian University of Technology in 2004 and PhD degree from Tianjin University in 2007, both of which are in Solid Mechanics. He is currently Associate Professor in the Department of Engineering Mechanics, Henan University of Technology, China. His main research interests are in computational mechanics and composite micromechanics. He has had more than 30 journal papers and three books published, respectively, by CRC Press and Tsinghua University Press. In 2010, He was awarded the Australia Endeavour Award.

Yi Xiao was born in China. She received her BS degree in Electronic and Telecommunication Engineering from The PLA University of Science and Technology, China, in 1984, and her PhD degree from The University of Sydney in computer vision and pattern recognition, in 2004. She is now Adjunct Research Fellow in the College of Engineering and Computer Science at the Australian National University.

Qing-Hua Qin received his BS degree in Mechanical Engineering from Chang An University, China, in 1982, and $\mathrm{MS}$ and $\mathrm{PhD}$ degrees from Huazhong University of Science and Technology (HUST), China, in 1984 and 1990, respectively, both in Applied Mechanics. After spending ten years lecturing at HUST, he was awarded a DAAD/K.C. Wong research fellowship in 1994 at the University of Stuttgart in Germany for nine months. From 1995-1997, he held a postdoctoral research fellowship position at Tsinghua University, China. Subsequently, he was awarded a Queen Elizabeth II fellowship in 1997 and a Professorial fellowship in 2002 at the University of Sydney by the Australian Research Council. He is currently working as Professor in the Research School of Engineering at the Australian National University, Australia. He was appointed guest professor at HUST in 2000 and was recipient of the J.G. Russell Award from the Australian Academy of Sciences. He has published over 250 journal papers and 7 monographs. 